\title{
PTPN1 promotes the progression of glioma by activating the MAPK/ERK and PI3K/AKT pathways and is associated with poor patient survival
}

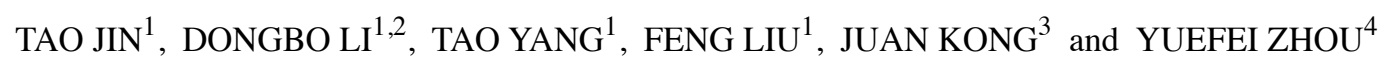 \\ ${ }^{1}$ Department of Neurosurgery, Ankang Central Hospital, Ankang, Shaanxi 725000; \\ ${ }^{2}$ The First Affiliated Hospital of Xi'an Jiaotong University School of Medicine, Xi'an, Shaanxi 710061; \\ ${ }^{3}$ Department of General Practice, Zhoukou Central Hospital, Zhoukou, Henan 466000; ${ }^{4}$ Department of Neurosurgery, \\ Xijing Hospital, Fourth Military Medical University, Xi'an, Shaanxi 710032, P.R. China
}

Received August 29, 2018; Accepted May 23, 2019

DOI: 10.3892/or.2019.7180

\begin{abstract}
Glioma is the most common primary brain tumor and is characterized by a poor prognosis. Protein tyrosine phosphatase 1B (PTPN1), as a non-transmembrane protein tyrosine phosphatase, has been reported to serve a critical role in different diseases, including cancer. However, the role of PTPN1 in the progression of glioma remains unclear. The present study investigated the expression and clinicopathological characteristics of PTPN1 by analyzing the data from The Cancer Genome Atlas and 136 patients with glioma. It was indicated that PTPN1 was overexpressed in glioma tissues and served as a predictor for poor prognosis in patients with glioma. In addition, a series of in vitro experiments were performed to examine the underlying mechanism of PTPN1 overexpression and the clinical prognosis in patients with glioma. Knockdown of PTPN1 by small interfering RNA suppressed proliferation of glioma cells, including SF295 and A172. In addition, cell mobility was also inhibited by PTPN1 knockdown, downregulating the expression of matrix metallopeptidase 2 (MMP-2) and MMP-9. As indicated by western blot analysis, the mitogen-activated protein kinase (MAPK)/extracellular-signal-regulated kinase (ERK) signaling pathway and the phosphatidylinositol 3-kinase (PI3K)/AKT serine/threonine kinase (AKT) signaling pathway was regulated by PTPN1, while knockdown of PTPN1 significantly suppressed the MAPK/ERK and PI3K/AKT pathways, in addition to the downstream oncogenic transcription factor MYC Proto-Oncogene. In conclusion, it was demonstrated that PTPN1 is upregulated in glioma tissue and the overexpression of PTPN1 predicted
\end{abstract}

Correspondence to: Dr Yuefei Zhou, Department of Neurosurgery, Xijing Hospital, Fourth Military Medical University, 15 ChangLe West Road, Xi'an, Shaanxi 710032, P.R. China

E-mail: ms375168@126.com

Key words: protein tyrosine phosphatase 1B, overexpression, glioma, prognosis, MAPK/ERK pathway, PI3K/AKT pathway the poor prognosis of patients with glioma. PTPN1 promotes the progression of glioma by activating the MAPK/ERK and PI3K/AKT pathways.

\section{Introduction}

Glioma is the most common form of aggressive central nervous system tumor in adults (1). Despite the fact that therapeutic strategies have advanced during recent years, patients have a very poor prognosis with a median survival of 15 months $(2,3)$. Genomic instability and signaling alternations are major contributors to radiotherapy and chemotherapy resistance of gliomas, therefore, leading to tumor recurrence following resection (4). Therefore, a better understanding of the molecular mechanisms in glioma tumorigenesis and an identification of novel prognostic molecular markers may potentially guide the design of functional, diagnostic and therapeutic strategies to this disease.

Protein tyrosine phosphatase 1B (PTPN1) is a non-transmembrane protein tyrosine phosphatase, which is located on the face of the endoplasmic reticulum, and serves a critical role in regulating the activities of major signaling pathways involved in different diseases, including obesity, diabetes and cancer $(5,6)$. However, to the best of our knowledge, the role of PTPN1 in tumor progression remains controversial (6). PTPN1 has been reported to be involved in the progression of different types of tumors by interacting with numerous oncogenic substrates, including SRC proto-oncogene, non-receptor tyrosine kinase (Src)/MAPK pathway in breast and lung cancer $(7,8)$. In addition, it has also been reported to negatively regulate several oncogenes, including Bcr-Abl and $\beta$-catenin $(9,10)$.

Previous studies have greatly extended our understanding of the molecular events of gliomagenesis, however, the function of PTPN1 in the development of gliomas is less understood.

\section{Materials and methods}

Bioinformatics. All the TCGA data were downloaded from the cancer Genome Browser of the UCSC database (https:/genome. 
ucsc.edu/index.html). Normalized PTPN1 mRNA expression in GBM and LGG with corresponding clinical data were included in files. In total, 10 non-neoplastic brain samples, 167 GBM samples and 530 LGG samples were analyzed.

Sample tissue collection. Paraffin-embedded samples, including 102 glioma specimens and 34 matched pairs of resected glioma tissues with non-neoplastic brain specimens, and fresh tissue, including 25 glioma specimens and 14 non-neoplastic brain specimens, were resected at the Xijing Hospital and the First Affiliated Hospital of Xi'an Jiaotong University School of Medicine (Xi'an, China) between 2008 and 2014. The present study was approved by the Ethics Committee of Xijing Hospital (Xi'an, China) in cooperation with the First Affiliated Hospital of Xi'an Jiaotong University School of Medicine. All patients underwent brain tumor resections and then were followed up until the death or the end of 2017. None of them had received radiotherapy or chemotherapy previously. Written informed consent was obtained from each patient prior to surgery. The clinicopathological characteristics and the follow-up information of the 136 patients, including sex, age, WHO grade, recurrence or not, receiving radiotherapy during the follow-up or not, receiving chemotherapy during the follow-up or not, the KPS score, the location of the tumor, living status at the end of the follow-up are presented in Table I. The median survival time of the glioma patients is provided in Table II. We could not provide the exact details of each individual since they will be used in future research from Xijing Hospital.

IHC analysis. Briefly, specimens were cut into sections (thickness, $5 \mu \mathrm{m}$ ). The sections were deparaffinized and rehydrated in a graded ethanol series, and washed in distilled water. The sections were then incubated with anti-PTPN1 antibody (dilution 1:200; cat. no. ab133244; Abcam, Cambridge, UK) overnight at $4^{\circ} \mathrm{C}$ and a secondary antibody (cat. no. sp9001; Beijing Zhongshan Jinqiao Biotechnology Co., Ltd., Beijing, China) for $30 \mathrm{~min}$. For visualization, diaminobenzidine (DAB; Beijing Zhongshan Jinqiao Biotechnology Co., Ltd.) was used. A light microscope (Olympus Corp.) was used to capture images to observe sample staining. To analyze the expression of PTPN1, the staining was categorized as previously described (11) and was confirmed by a pathologist at the First Affiliated Hospital of Xi'an Jiaotong University School of Medicine.

Copy number analysis. The copy number of PTPN1 was analyzed by reverse transcription-quantitative polymerase chain reaction (RT-qPCR) on a CFX384 Thermal CyclerDice ${ }^{\mathrm{TM}}$ (Bio-Rad Laboratories, Inc.) as previously described (12). This method has been well established and widely used in various types of human cancer (12-14). Specific primers and TaqMan probes were designed using Primer Express 3.0 (Applied Biosystems; Thermo Fisher Scientific, Inc.) to amplify PTPN1 and the internal reference gene $\beta$-actin. For the PTP1B gene, the TaqMan probe used was 5'-6FAM-TAACCCATCTCT GCCCTCTGATTCCTCAG-TAMRA-3', and the primers were 5'-GCCATTCATTTTCTCCAAAGTGA-3' (forward) and 5'-CGACCCGACTTCTAACTTCAGTGT-3' (reverse). For the $\beta$-actin gene, the probe was 5'-6-carboxyfluorescein-ATG
CCCTCCCCCATGCCATCC-tetramethyrhodamine-3', and the primers were 5'-TCACCCACACTGTGCCCATCTACGA-3' (forward) and 5'-TCGGTGAGGATCTTCATGAGGTA-3' (reverse). Using a PCR protocol previously described (15), the samples were run in triplicate, and $\beta$-actin was run in parallel, in order to normalize input DNA. Standard curves were established using serial dilutions of normal leukocyte DNA. PTPN1 amplification was defined by a copy number $\geq 4$.

Cell culture and siRNA transfection. Human glioma cell lines SF295 (cat. no. TCHu 58), A172 (cat. no. TCHu171) and glioblastoma cell line 'U87' of unknown origin (cat. no. TCHu138) were obtained and authenticated with STR profile in The Cell Bank of Type Culture Collection of the Chinese Academy of Sciences (www.cellbank.org.cn). Cells were cultured in DMEM media (cat. no. 10569010; Gibco; Thermo Fisher Scientific, Inc.) with $10 \%$ fetal bovine serum (FBS; Biological Industries) and maintained in an incubator with $5 \% \mathrm{CO}_{2}$ at $37^{\circ} \mathrm{C}$. For transient small interfering (si) RNA transfection, cells were transfected with siRNA targeting PTPN1 (si-PTPN1-1: GUC GGAUUAAACUACAUCATT, si-PTPN1-2: UGAUGUAGU UUAAUCCGACTT) and control siRNA (si-NC), constructed by Shanghai GenePharma Co., Ltd.

$R N A$ extraction and $R T-q P C R$. Total RNA was extracted from fresh samples and cell lines using TRIzol reagent (Takara Bio, Inc.) and cDNA was prepared using PrimeScript RT reagent kit (Roche Diagnostics), according to the manufacturer's protocols. RT-qPCR was performed on a CFX96 Thermal Cycler Dice ${ }^{\mathrm{TM}}$ real-time PCR system (Bio-Rad Laboratories, Inc.) using SYBR ${ }^{\mathrm{TM}}$ Green Master Mix (BioTools Pty. Ltd.) under the following cycling conditions: $3 \mathrm{~min}$ at $95^{\circ} \mathrm{C}$, followed by 35 cycles of $10 \mathrm{sec}$ at $95^{\circ} \mathrm{C}$ and $45 \mathrm{sec}$ at $58^{\circ} \mathrm{C}$. The mRNA expression of PTPN1 was normalized to $18 \mathrm{~S}$ rRNA. Relative mRNA expression was calculated by using $2^{-\Delta \Delta \mathrm{Cq}}$ method (16). Each sample was run in triplicate. Primers: 18s F: 5'-CGCCGCTAGAGGTGA AATTC-3', R: 5'-CTTTCGCTCTGGTCCGTCTT-3', PTPN1 F: 5'-GCCACCCAAACGAATCCT-3', R: 5'-CGACCCGAC TTCTAACTTCAG-3'.

Western blot analysis. Cells were lysed in pre-chilled RIPA buffer containing protease inhibitors (Sigma-Aldrich; Merck KGaA). Supernatants were collected and subjected to $10 \%$ SDS-PAGE with $200 \mathrm{ng}$ total protein, and transferred onto polyvinylidene fluoride (PVDF) membranes (Roche Diagnostics). The membranes were subsequently blocked with $5 \%$ non-fat dry milk for $1.5 \mathrm{~h}$ at room temperature. The membranes were incubated with primary antibodies, anti-PTPN1 (dilution 1:1,500; cat. no. ab133244; Epitomics; Abcam), anti-phospho-Akt (S473; dilution 1:1,000; cat. no. BS4007; Bioworld Technology, Inc.), anti-phospho-Akt (T308; dilution 1:1,000; cat. no. AP0056; Bioworld Technology, Inc.), anti-phospho-Erk1/2 (dilution 1:1,000; cat. no. 4370; Cell Signaling Technology, Inc.), anti-total-Akt (t-Akt, dilution 1:1,000; cat. no. BS1379; Bioworld Technology, Inc.), anti-total-Erk1/2 (t-Erk) (dilution 1:1,000; cat. no. 9102; Cell Signaling Technology, Inc.), anti-c-Myc (dilution 1:500; cat. no. sc-4084; Santa Cruz Biotechnology, Inc.), and anti-GAPDH (dilution 1:2,000; cat. no. AW5681; Abgent 
Table I. Clinicopathological characteristics of the glioma patients $(n=136)$.

\begin{tabular}{|c|c|c|}
\hline Characteristics & No. & Percent \\
\hline \multicolumn{3}{|l|}{ Sex } \\
\hline Male & 74 & 54.4 \\
\hline Female & 62 & 45.6 \\
\hline \multicolumn{3}{|l|}{ Age, years } \\
\hline Mean & 45.7 & \\
\hline SD & 16.4 & \\
\hline \multicolumn{3}{|l|}{ WHO grade } \\
\hline $\mathrm{I} / \mathrm{II}$ & 87 & 64.0 \\
\hline III/IV & 49 & 36.3 \\
\hline \multicolumn{3}{|l|}{ Recurrence } \\
\hline No & 39 & 28.7 \\
\hline Yes & 97 & 71.3 \\
\hline \multicolumn{3}{|l|}{ Radiotherapy } \\
\hline No & 57 & 41.9 \\
\hline Yes & 79 & 58.1 \\
\hline \multicolumn{3}{|l|}{ Chemotherapy } \\
\hline No & 81 & 59.6 \\
\hline Yes & 55 & 40.4 \\
\hline \multicolumn{3}{|l|}{ Seizures } \\
\hline No & 74 & 54.4 \\
\hline Yes & 62 & 45.6 \\
\hline \multicolumn{3}{|l|}{ KPS } \\
\hline$\leq 80$ & 56 & 41.2 \\
\hline$>80$ & 80 & 58.8 \\
\hline \multicolumn{3}{|l|}{ Location } \\
\hline Frontal lobe & 56 & 42.6 \\
\hline Temporal lobe & 35 & 25.7 \\
\hline Parietal lobe & 23 & 17.6 \\
\hline Occipital lobe & 8 & 5.9 \\
\hline Cerebellum & 6 & 4.4 \\
\hline Spinal cord & 2 & 1.5 \\
\hline Other part & 6 & 5.9 \\
\hline \multicolumn{3}{|l|}{ Survival status } \\
\hline Dead & 74 & 54.4 \\
\hline Alive & 62 & 45.6 \\
\hline
\end{tabular}

WHO, World Health Organization; KPS, Karnofsky Performance Score.

Biotech Co., Ltd.). This was followed by incubation with species-specific HRP-conjugated secondary antibodies (dilution 1:2,000; cat. nos. 130004 and 130023) from OriGene Technologies, Inc. Immunoblotting signals were visualized using the Western Bright enhanced chemiluminescence detection system (Advansta, Inc.).

Cell proliferation assay. Cells (1,000 cells/well) were seeded and cultured in 96-well plates for 1,3,5 and 7 days. At the indicated time-points, $20 \mu \mathrm{l}$ of $0.5 \mathrm{mg} / \mathrm{ml}$ MTT (Sigma-Aldrich; Merck KGaA) was added into the medium and incubated for
Table II. Mean and median survival time of various genotypes in glioma patients.

\begin{tabular}{lcc}
\hline Genotypes & Mean (month) & Median (month) \\
\hline PTPN1 $^{-}$ & 51.35 & 48.00 \\
PTPN1 $^{+}$ & 30.06 & 25.00 \\
\hline
\end{tabular}

$4 \mathrm{~h}$, followed by adding $150 \mu \mathrm{l} \mathrm{DMSO}$ for an additional $15 \mathrm{~min}$. A microplate reader (Dynatec Scientific Labs, Inc.) was used to measure the absorbance at a wavelength of $570 \mathrm{~nm}$.

Transwell migration assay. Cell migration and invasion assays were assessed by Transwell ( $8.0 \mu \mathrm{m}$ pore size; Corning Inc.). For the cell invasion assay, Transwell chambers were coated with Matrigel (4X dilution; $15 \mu \mathrm{l} /$ well; BD Biosciences). Cells were seeded in the upper chamber at a density of $1 \times 10^{4}$ cells $/ \mathrm{ml}$ for the migration assay and $1 \times 10^{4}$ cells $/ \mathrm{ml}$ for the invasion assay in $200 \mu \mathrm{l}$ of medium containing $0.5 \% \mathrm{FBS}$. The DMEM medium with $20 \%$ FBS $(1 \mathrm{ml})$ was added to the lower chamber. After 12 and $24 \mathrm{~h}$ of incubation, non-migrating/non-invading cells in the upper chamber were removed with a cotton swab, and migrating/invading cells were subsequently fixed in $100 \%$ methanol and stained with crystal violet solution ( $0.5 \%$ crystal violet in $2 \%$ ethanol) for $15 \mathrm{~min}$. Images were captured and five fields of each membrane were randomly selected. The number of migrating/invading cells was expressed as the average number of cells observed per microscopic (light microscope; Olympus Corp., Tokyo, Japan) field over the five fields.

Statistical analysis. Student's t-test was performed to compare two independent groups and two-way analysis of variance (ANOVA), with Bonferroni post hoc test was used for MTT group comparisons. One-way analysis of variance (ANOVA), with Dunnett's post hoc test was used for multiple group comparisons. A value of $\mathrm{P}<0.05$ was considered to indicate a statistically significant difference. Results are presented as the mean \pm standard deviation. One-way analysis of variance (ANOVA), with Dunnett's post hoc test was used for multiple comparisons between groups. The Kaplan-Meier survival curve and log-rank tests were used to assess the survival of patients with glioma. Cox regression analysis and logistic regression were used to evaluate the effects of PTPN1 copy number on survival and the odds ratio of each characteristic. Calculations and graphing were performed using SPSS (version 18.0; SPSS, Inc.) and GraphPad Prism (version 5.01; GraphPad Software, Inc., La Jolla, CA, USA).

\section{Results}

Amplification and overexpression of PTPN1 in human glioma. An analysis of PTPN1 expression pattern was performed using RT-qPCR, which indicated that PTPN1 was upregulated in glioma tissue compared with non-neoplastic brain tissues (Fig. 1A). Taking into consideration that gene amplification is a common mechanism for gene overexpression, an additional RT-qPCR was performed to analyze the copy number of the 

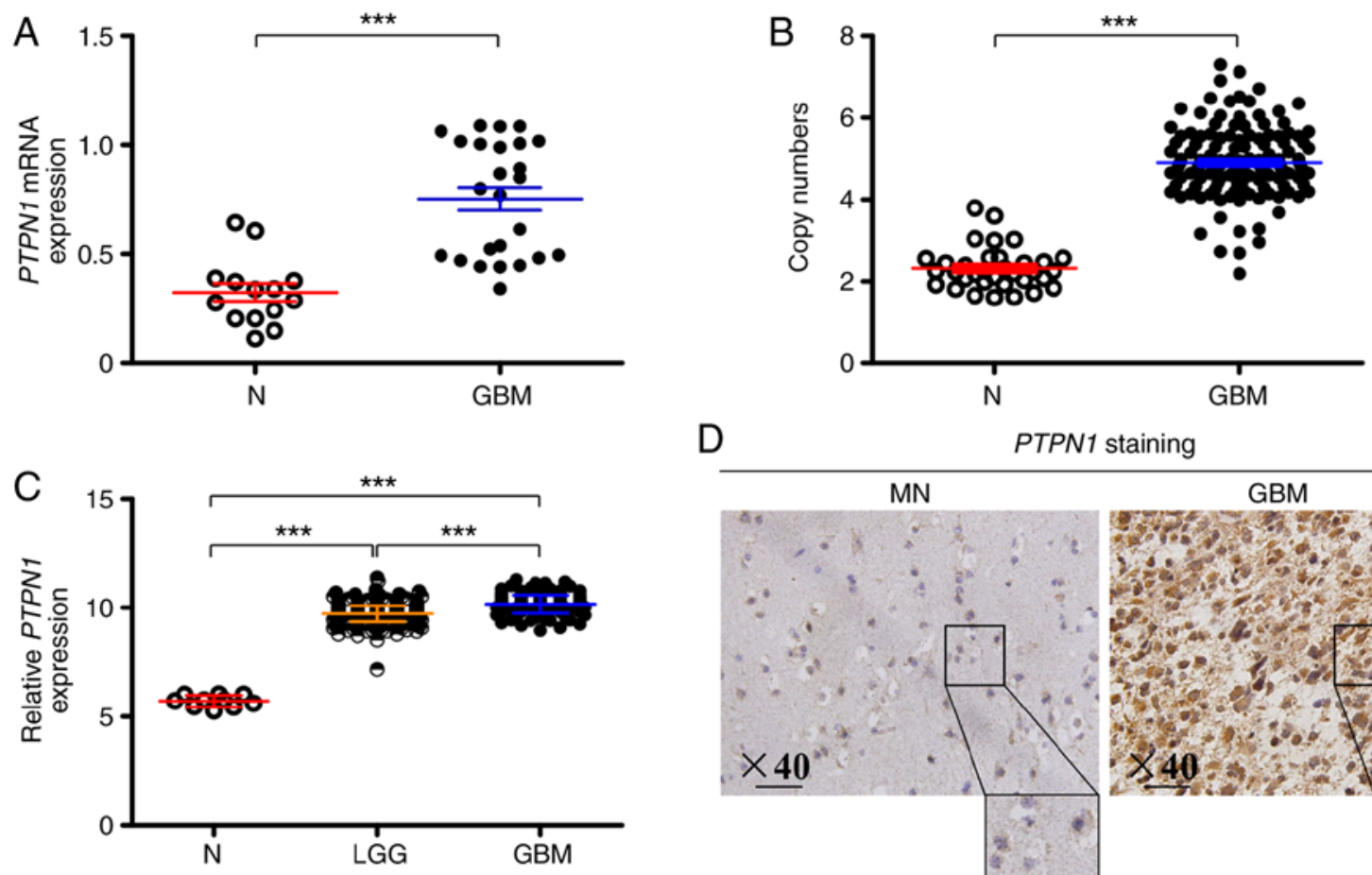

D

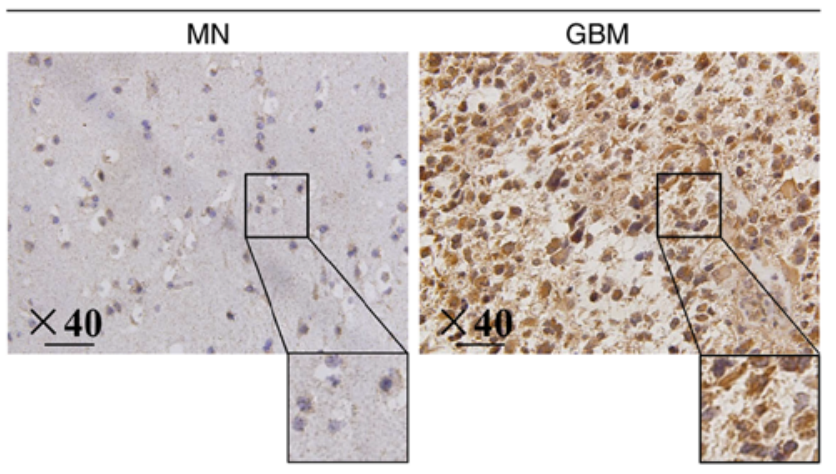

Figure 1. PTPN1 gene amplification and expression status in GBM. (A) PTPN1 mRNA expression was upregulated in 25 GBM specimens compared with 14 non-neoplastic brain specimens (N). (B) Relative gene copy number of PTPN1 in 136 GBM and 34 matched non-neoplastic brain specimens (N). (C) Expression of PTPN1 in GBM, LGG and non-neoplastic brain specimens (N) in TCGA database. (D) The expression of PTPN1 was higher in GBM tissue compared with $\mathrm{MN} ;{ }^{* * *} \mathrm{P}<0.001$. PTPN1, protein tyrosine phosphatase 1B; GBM, glioblastoma; LGG, low-grade gliomas; N, non-neoplastic brain specimens; MN, matched non-neoplastic brain tissue; TCGA, The Cancer Genome Atlas. Scale bar, $50 \mu \mathrm{m}$.

PTPN1 gene in 136 gliomas and 34 non-neoplastic brain specimens. As indicated in Fig. 1B, the copy number of the PTPN1 gene in glioma cases was significantly higher compared with non-neoplastic brain cases. Consistent with the RT-qPCR data, it was further confirmed that PTPN1 expression was significantly upregulated in low-grade gliomas (LGG) and was even higher in glioblastoma (GBM) compared with normal brain tissue, according to The Cancer Genome Atlas (TCGA) database (Fig. 1C). For protein expression, immunohistochemistry also confirmed that PTPN1 expression was increased in GBM tissue compared with non-neoplastic brain tissue (Fig. 1D).

PTPN1 amplification and overexpression is associated with poor prognosis in patients with glioma. To assess the effect of PTPN1 amplification on the survival of patients with glioma, Kaplan-Meier survival curves were used, grouped by the aberrant copy number of PTPN1, with the median copy number as the cut-off value. The data indicated that the patients with PTPN1 amplification had shorter mean and median survival times compared with the patients without PTPN1 amplification (Table II). The differences between the Kaplan-Meier survivals curves were also analyzed using the log-rank test. In accordance to the aforementioned, PTPN1 expression was significantly associated with poor survival among patients with GBM according to the TCGA database (Fig. 2). Univariate Cox regression analyses also indicated that there was a significant association of PTPN1 amplification with poor survival rate in patients with glioma (Table III). In addition, multivariate Cox regression analyses suggested that PTPN1 amplification was an independent risk

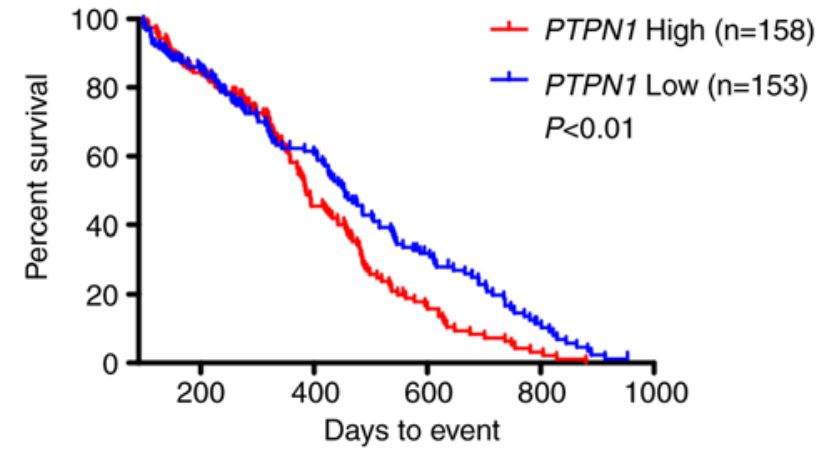

Figure 2. Association of PTPN1 high expression with poor survival of patients with glioma from TCGA database. Patients with high PTPN1 expression exhibited shorter survival times compared with patients with low PTPN1 expression. Kaplan-Meier survival curves were used to assess the survival of GBM patients. Median expression level was used as the cutoff point. PTPN1, protein tyrosine phosphatase 1B; TCGA, The Cancer Genome Atlas; GBM, glioblastoma.

factor for patients with glioma with respect to copy number, pathological diagnosis, World Health Organization (WHO), and relapse (Table III). In addition, PTPN1 amplification was associated with a significantly increased risk of tumor relapse and age (Table IV).

PTPN1 knockdown inhibits glioma cell growth. Increased expression of PTPN1 in glioma indicated that PTPN1 may be a putative oncogene in primary glioma. Firstly, we assessed the expression of PTPN1 in different glioma cells. As revealed 
Table III. Prognostic value of clinicopathological factors and PTPN1 amplification using univariate and multivariate Cox regression analysis $(\mathrm{n}=136)$.

\begin{tabular}{|c|c|c|c|c|}
\hline \multirow[b]{2}{*}{ Variable } & \multicolumn{2}{|c|}{ Univariate analysis } & \multicolumn{2}{|c|}{ Multivariate analysis } \\
\hline & Hazard Ratio & P-value & Hazard Ratio & P-value \\
\hline Copy number & $1.88(1.32-3.10)$ & $0.01^{\mathrm{c}}$ & $1.69(1.04-2.73)$ & $0.03^{\mathrm{c}}$ \\
\hline Pathological diagnosis ${ }^{\mathrm{a}}$ & $2.31(1.74-2.68)$ & $<0.01^{\mathrm{d}}$ & $2.03(1.59-2.59)$ & $<0.01^{\mathrm{d}}$ \\
\hline $\mathrm{WHO}^{\mathrm{b}}$ & $4.03(2.61-6.39)$ & $<0.01^{\mathrm{d}}$ & $3.82(2.40-6.08)$ & $<0.01^{\mathrm{d}}$ \\
\hline KPS & $1.25(0.86-1.97)$ & 0.51 & $1.11(0.71-1.74)$ & 0.64 \\
\hline Relapse & $4.75(2.35-7.95)$ & $<0.01^{\mathrm{d}}$ & $4.10(2.21-7.61)$ & $<0.01^{\mathrm{d}}$ \\
\hline
\end{tabular}

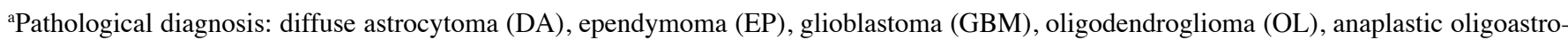
cytoma (AOA). ${ }^{b} \mathrm{WHO}$ grade (I, II, III; IV). ${ }^{\mathrm{c}} \mathrm{P}<0.05 ;{ }^{\mathrm{d}} \mathrm{P}<0.01$
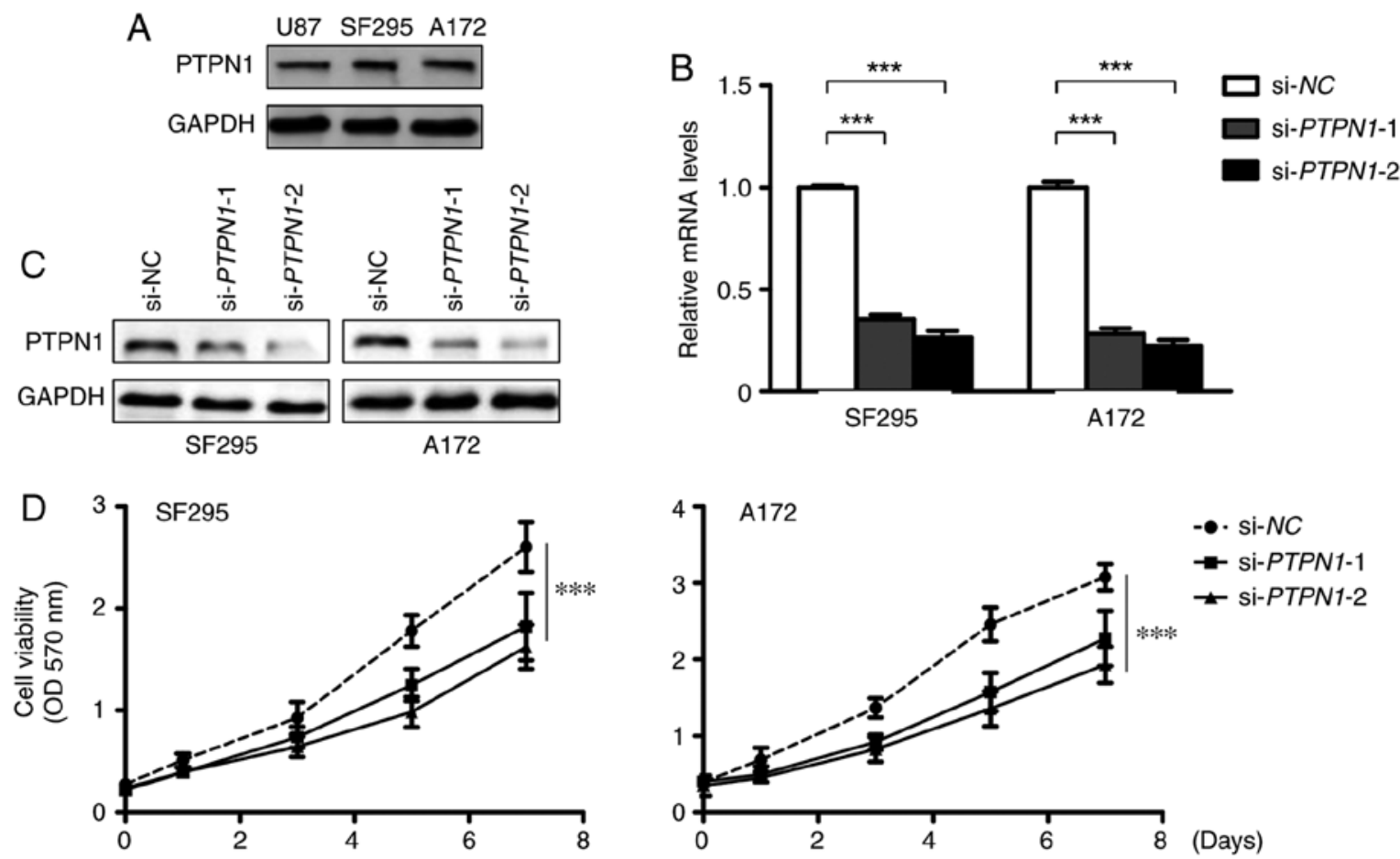

Figure 3. PTPN1 expression knockdown in glioma cell lines SF295 and A172, and MTT array. (A) Basic expression levels of PTPN1 in U87, SF295 and A172 were determined by western blot analysis. SF295 and A172 cells were transfected with (B) non-sense control siRNA (si-NC) or two different PTPN1 specific siRNAs, (si-PTPN1-1, si-PTPN1-2) and (C) western blot analysis was performed.(D) PTPN1 knockdown inhibited glioma cell proliferation. Experiments were performed in triplicate. ${ }^{* * *} \mathrm{P}<0.001$. PTPN1, protein tyrosine phosphatase $1 \mathrm{~B}$; si, small interfering.

in Fig. 3A, glioma cell lines SF295 and A172 exhibited higher PTPN1 expression than U87, this may due to the different genetic backgrounds of these cell lines. Thus, the effect of PTPN1 on glioma cell growth was investigated by knocking down PTPN1 in SF295 and A172 glioma cells. The knockdown of PTPN1 expression using two different siRNA sequences, si-PTPN1-1 and 2, was confirmed by RT-qPCR and western blot analysis (Fig. 3B and C). Glioma cell proliferation was reduced when PTPN1 expression was inhibited by specific PTPN1 siRNA compared with control siRNA (si-NC), in particular si-PTPN1-2 (Fig. 3D).

Downregulated PTPN1 expression inhibits glioma cell migration and invasion. Due to the fact that invasion and metastasis are the main causes of cancer-associated mortality (17), the aim of the present study was to examine the effect of PTPN1 knockdown on cell migration and invasion using Transwell assays. Downregulated PTPN1 expression decreased the number of migrated cells (Fig. 4A). In addition, PTPN1 knockdown also reduced the number of cells passing through the Matrigel-coated membrane (Fig. 4B). In addition, RT-qPCR analysis indicated that the decreased metastasis-associated phenotypes were due to the reduced expression of MMPs, which are involved in cancer cell metastasis (18), in glioma cells (Fig. 4C). Therefore, the inhibition of epithelial-mesenchymal transition (EMT) process by PTPN1 downregulation may contribute to the suppression of glioma cell metastasis (Fig 4D). 
A

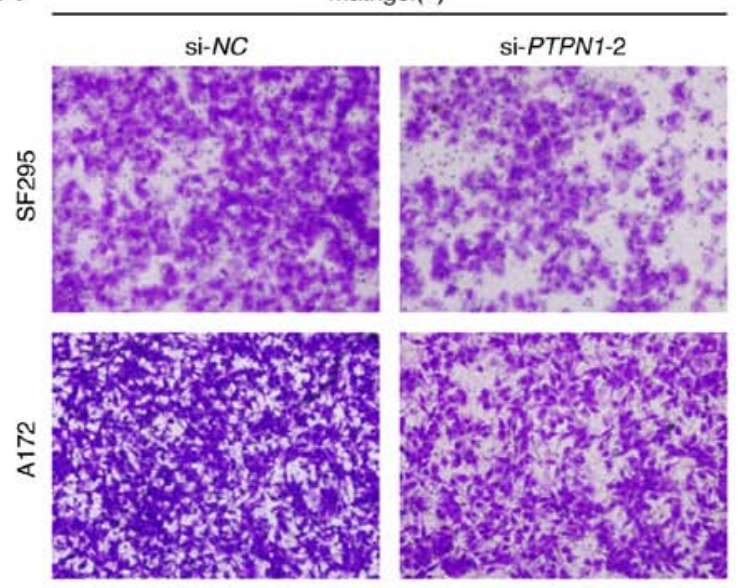

B
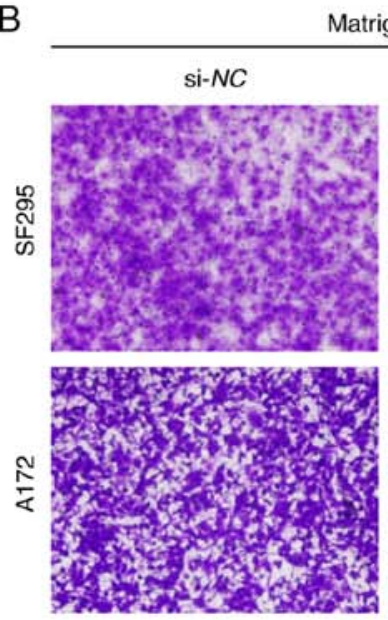

Matrigel(+)

si-PTPN1-2

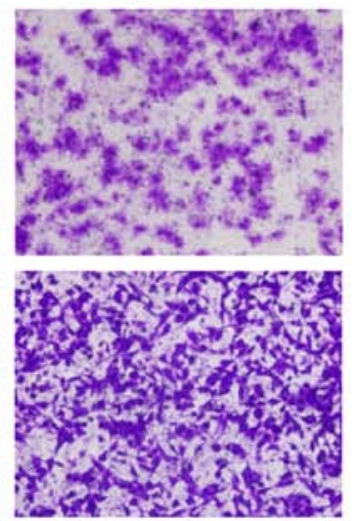

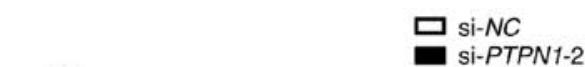
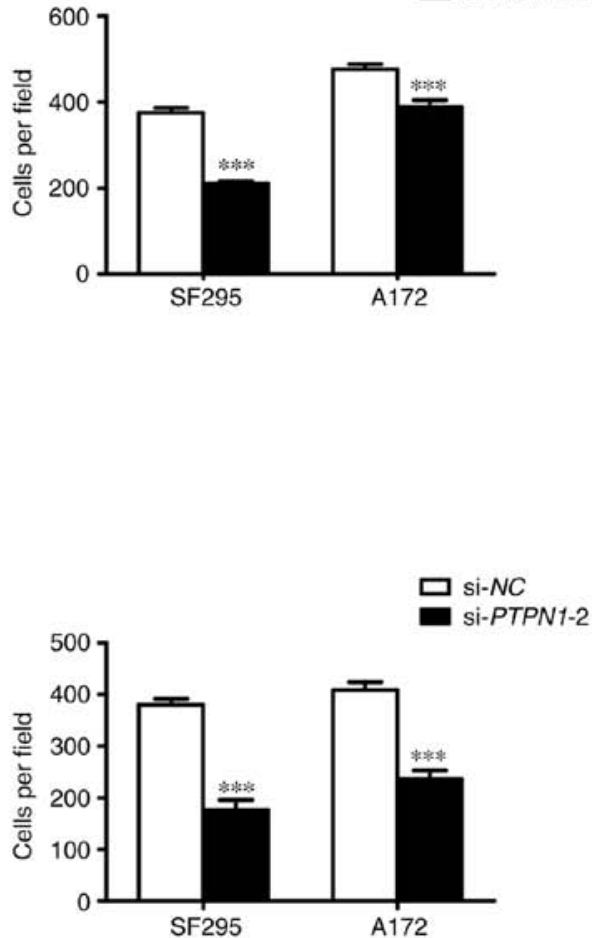
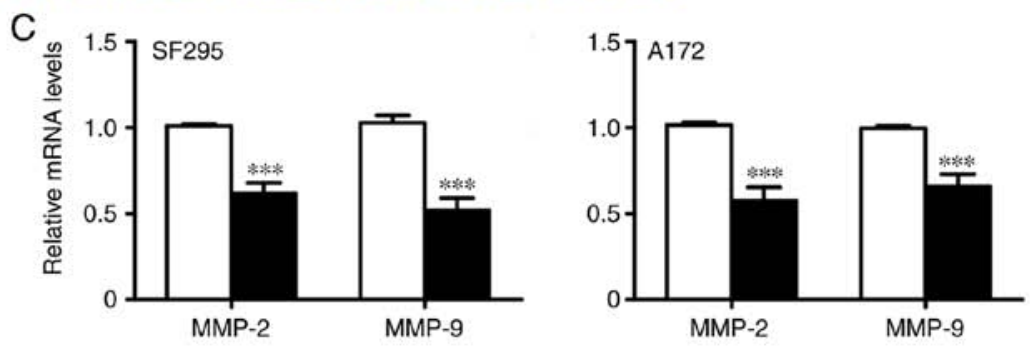

$\square$ si-NC

si-PTPN1-2
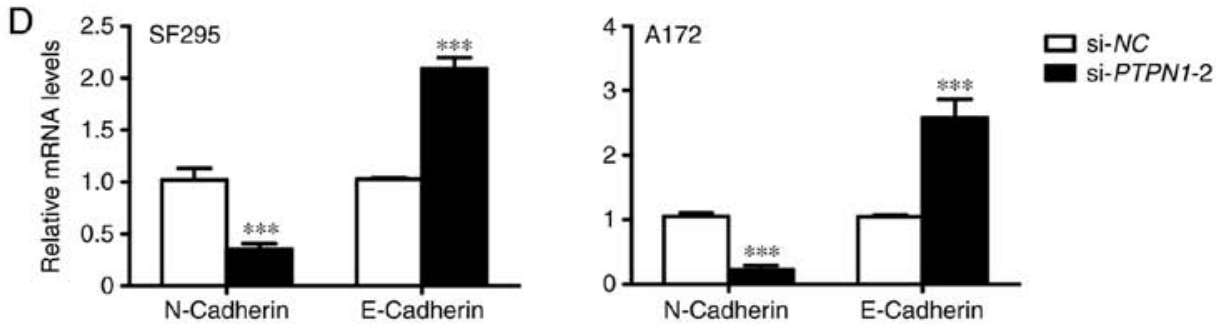

Figure 4. Downregulation of PTPN1 expression inhibits glioma cell migration and invasion in SF295 and A172 glioma cells. (A) Migration and (B) invasion of SF295 and A172 glioma cells with PTPN1 knockdown. Representative fields are presented on the left. (C) PTPN1 knockdown suppressed the expression of EMT-associated genes MMP-2 and MMP-9. (D) The expression of E-cadherin and N-cadherin and the effect of PTPN1 knockdown on the process of EMT. Expression levels of these genes were normalized with $18 \mathrm{~S}$ rRNA levels. ${ }^{* * *} \mathrm{P}<0.001$. PTPN1, protein tyrosine phosphatase 1B; MMP-2/9, matrix metallopeptidase 2/9, EMT, epithelial-mesenchymal transition.

PTPN1 regulates the MAPK/ERK and PI3K/AKT signaling pathways in glioma. Previous studies have reported that the MAPK/ERK pathway and its downstream Myc serve an important role in the development and progression of glioma $(19,20)$. Therefore, the present study investigated the effect of PTPN1 on the activities of the MAPK/ERK pathway. As indicated in Fig. 5,
PTP1N knockdown suppressed phosphorylation of MAPK/ERK pathway functional kinase ERK1/2 and the expression of ERK downstream c-Myc. In addition, the PI3K/AKT pathway has been reported to regulate glioma metastasis $(21,22)$. PTPN1 expression silencing also suppressed the phosphorylation of AKT in Thr308 and Ser473 (Fig. 5). These results indicated that 
Table IV. Association of PTPN1 copy number with clinicopathological characteristics in patients with glioma (OR and 95\% CI).

\begin{tabular}{llc}
\hline & \multicolumn{2}{c}{ PTPN1 copy number } \\
\cline { 2 - 3 } Characteristics & OR $^{\mathrm{a}}(95 \% \mathrm{CI})$ & P-value \\
\hline Pathological diagnosis $^{\mathrm{b}}$ & $0.38(0.12-1.48)$ & 0.86 \\
Age & $0.52(0.30-0.90)$ & $0.02^{\mathrm{f}}$ \\
Sex & $1.43(0.71-2.87)$ & 0.31 \\
WHO grade $^{\mathrm{c}}$ & $1.34(0.65-2.75)$ & 0.42 \\
Recurrence $_{\text {KPS score }}^{\mathrm{d}}$ & $2.35(1.59-5.49)$ & $0.03^{\mathrm{f}}$ \\
Survival status $^{\mathrm{e}}$ & $1.07(0.54-2.14)$ & 0.85 \\
\hline
\end{tabular}

${ }^{\mathrm{a}} \mathrm{OR}$, Odds ratio with $95 \%$ confidence interval (CI). ${ }^{\mathrm{b}}$ Pathological diagnosis: Diffuse astrocytoma (DA), ependymoma (EP), glioblastoma (GBM), oligodendroglioma (OL), anaplastic oligoastrocytoma (AOA). ${ }^{c}$ WHO grade (I, II, III; IV). ${ }^{d} \mathrm{KPS}(>80 ; \leq 80)$. ${ }^{\mathrm{e} S u r v i v a l}$ status (alive vs. dead). ${ }^{\mathrm{f}} \mathrm{P}<0.05$. Age $(>45 ; \leq 45)$.
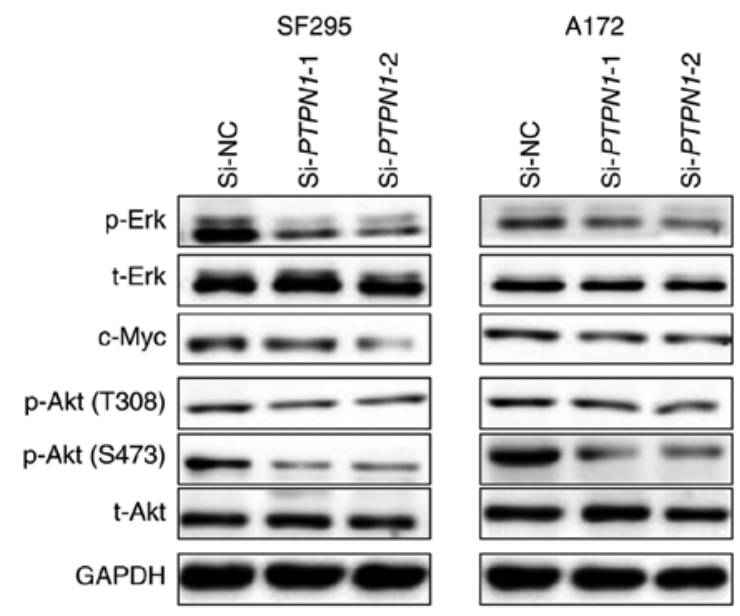

Figure 5. Effects of PTPN1 knockdown on the MAPK/ERK and PI3K/AKT pathways in SF295 and A172 glioma cells. PTPN1 functions as a potential oncogene by activating the MAPK and PI3K/AKT signaling pathways. Experiments were performed $>3$ times. PTPN1, protein tyrosine phosphatase 1B; MAPK, mitogen-activated protein kinase; ERK, extracellular-signal-regulated kinase; PI3K, phosphatidylinositol 3-kinase; AKT, AKT serine/threonine kinase.

PTPN1 promotes glioma cell proliferation and metastasis by activating the MAPK/ERK and PI3K/AKT signaling pathways.

\section{Discussion}

PTPN1, as an oncogene, has been reported as a potential therapeutic target and prognostic marker in breast cancer and gastric cancer $(23,24)$, however its role in glioma has yet to be established. Studies involving the underlying mechanism of PTPN1 in gliomagenesis have reported controversial findings on the role of PTPN1: a number of previous studies have reported PTPN1 as a potential tumor suppressor gene in glioma $(25,26)$, while other studies have suggested PTPN1 to serve an oncogenic role in glioma $(27,28)$. The results of the present study provided a series of evidence in support of an oncogenic role for PTPN1 in glioma. Furthermore, high expression of PTPN1 predicted poor prognosis of patients with GBM, therefore, indicating that PTPN1 may serve as a prognostic marker and a putative target in the treatment of glioma.

A previous study indicated that PTPN1 overexpression may activate Src by reducing phosphorylation at tyrosine 530 (29). Src activation has been reported to be associated with proliferation, survival and metastasis in cancer cells, by stimulating multiple signaling pathways, including the Src/PI3K/Akt and Src/MAPK/ERK signaling pathways (30). The MAPK/ERK and PI3K/Akt signaling pathways have been reported to serve an important role in the proliferation of numerous types of cancer cells (31-33). EGFR, as an upstream of the MAPK/ERK and PI3K/AKT signaling pathways, has been reported to be overexpressed in glioma cells, resulting in proliferation with downstream effects $(34,35)$. In addition, the activation of the MAPK/ERK pathway has been revealed to promote the expression of vascular endothelial growth factor, which is an important angiogenic factor for glioma (36). An increasing number of studies have reported that the hyperactivation of AKT is involved in the migration and invasion of tumor cells $(37,38)$. In addition, a previous study confirmed that abnormal activation of the PI3K/AKT signaling pathway may induce overexpression of oncogene sprouty RTK signaling antagonist 1, leading to inhibited apoptosis and increased proliferation in human glioma cells (39). Therefore, it was hypothesized that overexpression of PTPN1 may lead to glioma progression and metastasis by activating the Src/PI3K/AKT and Src/MAPK/ERK signaling pathways, and may predict poor prognosis of patients with glioma. The data of the present study indicated that PTPN1 silencing inhibits phosphorylation of ERK and AKT in glioma cells. In addition, PTPN1 knockdown inhibited the EMT process, which has been demonstrated to serve a key role during the early steps of invasion and metastasis of epithelial malignancies (40), by increasing the expression of epithelial cell marker E-cadherin and reducing the expression of mesenchymal marker $\mathrm{N}$-cadherin. These results indicated that downregulation of PTPN1 may contribute to the inhibition of glioma cell migration and invasion. These insights into the molecular mechanisms may provide potential treatment options for a subset of cancers, including glioma, via inhibition of PTPN1.

Notably, PTPN1 may play an important role in the transformation of LGG to GBM, which was supported by analyzing LGG and GBM data in TCGA database. In fact, the expression of certain biomarkers undergo change when the progression of LGG to GBM occurs (41). However, we did not have enough LGG patient samples or cell lines to verify our hypothesis. Thus, further studies are required to investigate the role of PTPN1 in the conversion of LGG to GBM. In addition, for comparisons with SF295 and A172, we did not have an appropriate non-cancerous negative control since we failed to have a non-malignant glial cell line in our in vitro study, and this was also a limitation of this study.

In summary, the present study demonstrated PTPN1 is overexpressed in glioma compared with matched normal brain tissue by immunohistochemistry (IHC). In addition, PTPN1 overexpression was associated with the poor survival of patients. PTPN1 knockdown was indicated to suppress glioma cell growth, migration and invasion. Therefore, PTPN1 was 
revealed to promote glioma cell proliferation and invasion through the MAPK/ERK and PI3K/AKT pathways. The data of the present study, to the best of our knowledge, was the first to verify the oncogenic role of PTPN1 in glioma tumorigenesis and investigate the molecular mechanism of PTPN1 in the promotion of glioma cell proliferation and survival. Overexpression of PTPN1 has been reported to be associated with shorter survival of patients and glioma metastasis. Therefore, PTPN1 may serve as a potential prognostic marker and a possible therapeutic target against glioma.

\section{Acknowledgements}

This research was supported by the First Affiliated Hospital of Xi'an Jiaotong University School of Medicine and Xijing Hospital of the Fourth Military Medical University (the First Affiliated Hospital of Fourth Military Medical University).

\section{Funding}

No funding was received.

\section{Availability of data and materials}

The datasets used during the current study are available from the corresponding author on reasonable request.

\section{Authors' contributions}

JK and YZ designed the research and approved the final version of the manuscript to be published. TJ as well as DL analyzed the data and wrote the manuscript. TJ, TY and FL conducted the research. All authors read and approved the manuscript and agree to be accountable for all aspects of the research in ensuring that the accuracy or integrity of any part of the work are appropriately investigated and resolved.

\section{Ethics approval and consent to participate}

Written informed consent was obtained from all of the patients before the surgery. The present study was approved by the Ethics Committee of Ankang Central Hospital, First Affiliated Hospital of Xi'an Jiaotong University and Xijing Hospital.

\section{Patient consent for publication}

Identifying information, including names, initials, date of birth or hospital numbers, images or statements were not included in the manuscript. Written informed consent was obtained from all of the patients prior to treatment.

\section{Competing interests}

The authors declare that they have no competing interests.

\section{References}

1. Wen PY, Macdonald DR, Reardon DA, Cloughesy TF, Sorensen AG, Galanis E, Degroot J, Wick W, Gilbert MR, Lassman AB, et al: Updated response assessment criteria for high-grade gliomas: Response assessment in neuro-oncology working group. J Clin Oncol 28: 1963-1972, 2010.
2. Mittal S, Pradhan S and Srivastava T: Recent advances in targeted therapy for glioblastoma. Expert Rev Neurother 15: 935-946, 2015.

3. Wen PY and Kesari S: Malignant gliomas in adults. N Engl J Med 359: 492-507, 2008.

4. Huse JT and Holland EC: Targeting brain cancer: Advances in the molecular pathology of malignant glioma and medulloblastoma. Nat Rev Cancer 10: 319-331, 2010.

5. Seely BL, Staubs PA, Reichart DR, Berhanu P, Milarski KL, Saltiel AR, Kusari J and Olefsky JM: Protein tyrosine phosphatase 1B interacts with the activated insulin receptor. Diabetes 45 : 1379-1385, 1996.

6. Lessard L, Stuible M and Tremblay ML: The two faces of PTP1B in cancer. Biochim Biophys Acta 1804: 613-619, 2010.

7. Cortesio CL, Chan KT, Perrin BJ, Burton NO, Zhang S, Zhang ZY and Huttenlocher A: Calpain 2 and PTP1B function in a novel pathway with Src to regulate invadopodia dynamics and breast cancer cell invasion. J Cell Biol 180: 957-971, 2008.

8. Liu H, Wu Y, Zhu S, Liang W, Wang Z, Wang Y, Lv T, Yao Y, Yuan D and Song Y: PTP1B promotes cell proliferation and metastasis through activating src and ERK1/2 in non-small cell lung cancer. Cancer Lett 359: 218-225, 2015.

9. LaMontagne KR Jr., Hannon G and Tonks NK: Protein tyrosine phosphatase PTP1B suppresses p210 bcr-abl-induced transformation of rat-1 fibroblasts and promotes differentiation of K562 cells. Proc NatI Acad Sci USA 95: 14094-14099, 1998.

10. Balsamo J, Leung T, Ernst H, Zanin MK, Hoffman S and Lilien J: Regulated binding of PTP1B-like phosphatase to N-cadherin: Control of cadherin-mediated adhesion by dephosphorylation of beta-catenin. J Cell Biol 134: 801-813, 1996.

11. Chen H, Chen W, Zhang X, Hu L, Tang G, Kong J and Wang Z: E26 transformation (ETS)-specific related transcription factor-3 (ELF3) orchestrates a positive feedback loop that constitutively activates the MAPK/Erk pathway to drive thyroid cancer. Oncol Rep 41: 570-578, 2019.

12. Wu G, Mambo E, Guo Z, Hu S, Huang X, Gollin SM, Trink B, Ladenson PW, Sidransky D and Xing M: Uncommon mutation, but common amplifications of the PIK3CA gene in thyroid tumors. J Clin Endocrinol Metab 90: 4688-4693, 2005.

13. Engelman JA, Zejnullahu K, Mitsudomi T, Song Y, Hyland C, Park JO, Lindeman N, Gale CM, Zhao X, Christensen J, et al: MET amplification leads to gefitinib resistance in lung cancer by activating ERBB3 signaling. Science 316: 1039-1043, 2007.

14. Kawano O, Sasaki H, Okuda K, Yukiue H, Yokoyama T, Yano M and Fujii Y: PIK3CA gene amplification in Japanese non-small cell lung cancer. Lung Cancer 58: 159-160, 2007.

15. Mambo E, Gao X, Cohen Y, Guo Z, Talalay P and Sidransky D: Electrophile and oxidant damage of mitochondrial DNA leading to rapid evolution of homoplasmic mutations. Proce Natl Acad Sci USA 100: 1838-1843, 2003.

16. Livak KJ and Schmittgen TD: Analysis of relative gene expression data using real-time quantitative PCR and the 2(-Delta Delta C(T)) method. Methods 25: 402-408, 2001.

17. Jemal A, Bray F, Center MM, Ferlay J, Ward E and Forman D: Global cancer statistics. CA Cancer J Clin 61: 69-90, 2011.

18. Egeblad M and Werb Z: New functions for the matrix metalloproteinases in cancer progression. Nat Rev Cancer 2: 161-174, 2002.

19. Guha A, Feldkamp MM, Lau N, Boss G and Pawson A: Proliferation of human malignant astrocytomas is dependent on Ras activation. Oncogene 15: 2755-2765, 1997.

20. Annibali D, Whitfield JR, Favuzzi E, Jauset T, Serrano E, Cuartas I, Redondo-Campos S, Folch G, Gonzàlez-Juncà A, Sodir NM, et al: Myc inhibition is effective against glioma and reveals a role for Myc in proficient mitosis. Nat Commun 5: 4632, 2014.

21. Tu M, Wange W, Cai L, Zhu P, Gao Z and Zheng W: IL-13 receptor alpha2 stimulates human glioma cell growth and metastasis through the $\mathrm{Src} / \mathrm{PI} 3 \mathrm{~K} / \mathrm{Akt} / \mathrm{mTOR}$ signaling pathway. Tumour Biol 37: 14701-14709, 2016.

22. Zhang X, Chen T, Zhang J, Mao Q, Li S, Xiong W, Qiu Y, Xie Q and Ge J: Notch1 promotes glioma cell migration and invasion by stimulating beta-catenin and NF-kappaB signaling via AKT activation. Cancer Sci 103: 181-190, 2012.

23. Taliaferro-Smith L, Nagalingam A, Knight BB, Oberlick E, Saxena NK and Sharma D: Integral role of PTP1B in adiponectinmediated inhibition of oncogenic actions of leptin in breast carcinogenesis. Neoplasia 15: 23-38, 2013.

24. Wang J, Liu B, Chen X, Su L, Wu P, Wu J and Zhu Z: PTP1B expression contributes to gastric cancer progression. Med Oncol 29: 948-956, 2012. 
25. Reichardt W, Jung V, Brunner C, Klein A, Wemmert S, Romeike BF, Zang KD and Urbschat S: The putative serine/threonine kinase gene STK15 on chromosome 20q13.2 is amplified in human gliomas. Oncol Rep 10: 1275-1279, 2003.

26. Mondol AS, Tonks NK and Kamata T: Nox4 redox regulation of PTP1B contributes to the proliferation and migration of glioblastoma cells by modulating tyrosine phosphorylation of coronin-1C. Free Radic Biol Med 67: 285-291, 2014.

27. Akasaki Y, Liu G, Matundan HH, Ng H, Yuan X, Zeng Z, Black KL and Yu JS: A peroxisome proliferator-activated receptor-gamma agonist, troglitazone, facilitates caspase- 8 and -9 activities by increasing the enzymatic activity of protein-tyrosine phosphatase-1B on human glioma cells. J Biol Chem 281: 6165-6174, 2006.

28. Petri MK, Koch P, Stenzinger A, Kuchelmeister K, Nestler U, Paradowska A, Steger K, Brobeil A, Viard M and Wimmer M PTPIP51, a positive modulator of the MAPK/Erk pathway, is upregulated in glioblastoma and interacts with 14-3-3 $\beta$ and PTP1B in situ. Histol Histopathol 26: 1531-1543, 2011.

29. Zhu S, Bjorge JD and Fujita DJ: PTP1B contributes to the oncogenic properties of colon cancer cells through Src activation. Cancer Res 67: 10129-10137, 2007.

30. Chen T, George JA and Taylor CC: Src tyrosine kinase as a chemotherapeutic target: Is there a clinical case? Anticancer Drugs 17: 123-131, 2006.

31. Wang J, Zhang Z, Li R, Mao F, Sun W, Chen J, Zhang H, Bartsch JW, Shu K and Lei T: ADAM12 induces EMT and promotes cell migration, invasion and proliferation in pituitary adenomas via EGFR/ERK signaling pathway. Biomed Pharmacother 97: 1066-1077, 2018.

32. Du MR, Zhou WH, Yan FT, Zhu XY, He YY, Yang JY and Li DJ: Cyclosporine A induces titin expression via MAPK/ERK signalling and improves proliferative and invasive potential of human trophoblast cells. Hum Reprod 22: 2528-2537, 2007.
33. Huang HY, Chang HF, Tsai MJ, Chen JS and Wang MJ: 6-Mercaptopurine attenuates tumor necrosis factor-alpha production in microglia through Nur77-mediated transrepression and PI3K/Akt/mTOR signaling-mediated translational regulation. J Neuroinflammation 13: 78, 2016.

34. Butowski NA and Chang SM: Glial tumors: The current state of scientific knowledge. Clin Neurosurg 53: 106-113, 2006.

35. Purow BW, Sundaresan TK, Burdick MJ, Kefas BA, Comeau LD, Hawkinson MP, Su Q, Kotliarov Y, Lee J, Zhang W and Fine HA: Notch-1 regulates transcription of the epidermal growth factor receptor through p53. Carcinogenesis 29: 918-925, 2008.

36. Guo G, Yao W, Zhang Q and Bo Y: Oleanolic acid suppresses migration and invasion of malignant glioma cells by inactivating MAPK/ERK signaling pathway. PLoS One 8: e72079, 2013.

37. Hollander MC, Blumenthal GM and Dennis PA: PTEN loss in the continuum of common cancers, rare syndromes and mouse models. Nat Rev Cancer 11: 289-301, 2011.

38. Pao W and Girard N: New driver mutations in non-small-cell lung cancer. Lancet Oncol 12: 175-180, 2011.

39. Chai C, Song LJ, Han SY, Li XQ and Li M: MicroRNA-21 promotes glioma cell proliferation and inhibits senescence and apoptosis by targeting SPRY1 via the PTEN/PI3K/AKT signaling pathway. CNS Neurosci Ther 24: 369-380, 2018

40. Jiang L, Wang Z, Liu C, Gong Z, Yang Y, Kang H, Li Y and $\mathrm{Hu}$ G: TrkB promotes laryngeal cancer metastasis via activation PI3K/AKT pathway. Oncotarget 8: 108726-108737, 2017.

41. Ceccarelli M, Barthel FP, Malta TM, Sabedot TS, Salama SR, Murray BA, Morozova O, Newton Y, Radenbaugh A, Pagnotta SM, et al: Molecular profiling reveals biologically discrete subsets and pathways of progression in diffuse glioma. Cell 164: 550-563, 2016 